\title{
The Hospital Anxiety and Depression Scale: low sensitivity for depression screening in demented and non-demented hospitalized elderly
}

\author{
Nikolaos Samaras, ${ }^{1}$ François R. Herrmann, ${ }^{1}$ Dimitrios Samaras, ${ }^{2}$ \\ Pierre-Olivier Lang, ${ }^{3,4}$ Alessandra Canuto, ${ }^{5}$ Alexandre Forster, ${ }_{1}{ }^{2}$ \\ Henriette Hilleret ${ }^{6}$ and Gabriel Gold ${ }^{7}$ \\ ${ }^{1}$ Department of Internal Medicine, Rehabilitation and Geriatrics, Geneva University Hospitals, Geneva, Switzerland \\ ${ }^{2}$ Department of Medical Specialties, Clinical Nutrition, Geneva University Hospitals, Geneva, Switzerland \\ ${ }^{3}$ Nescens Center of Preventive Medicine, Clinic of Genolier, Genolier, Switzerland \\ ${ }^{4}$ Translational Medicine Research Group, Cranfield Health, Cranfield University, Cranfield, UK \\ ${ }^{5}$ Service of Psychiatry of Liaison and Crisis Intervention, Geneva University Hospitals and Faculty of Medicine of the University of Geneva, Geneva, \\ Switzerland \\ ${ }^{6}$ Service of Psychiatry of Liaison and Crisis Intervention, Geneva University Hospitals, Geneva, Switzerland \\ ${ }^{7}$ Department of Internal Medicine Rehabilitation and Geriatrics, Geneva University Hospitals and Faculty of Medicine of the University of Geneva, \\ Geneva, Switzerland
}

\section{ABSTRACT}

Background: We currently use the depression subscale (HADD) of the Hospital Anxiety and Depression Scale (HADS) for depression screening in elderly inpatients. Given recent concerns about the performance of the HADD in this age group, we performed a quality-control study retrospectively comparing HADD with the diagnosis of depression by a psychiatrist. We also studied the effect of dementia on the scale's performance.

Methods: HADS produces two 7-item subscales assessing depression or anxiety. The HADD was administered by a neuropsychologist. As "gold standard" we considered the psychiatrist's diagnosis based on the Diagnostic and Statistical Manual of Mental Disorders, Fourth Edition, criteria. Patients older than 65 years, assessed by both the HADD and the psychiatrist, with a clinical dementia rating (CDR) score lower than 3, were included. The effect of dementia was assessed by forming three groups according to the CDR score (CDR0-0.5, CDR1, and CDR2). Simple and multiple logistic regression models were applied to predict the psychiatrist's depression diagnosis from HADD scores. Areas under the receiver operating characteristics curve (AUC) were plotted and compared by $\chi^{2}$ tests.

Results: On both univariate and multiple analyses, HADD predicted depression diagnosis but performed poorly (univariate: $\mathrm{p}=0.009, \mathrm{AUC}=0.60$ (95\% confidence interval $(\mathrm{CI})=0.53-0.66)$; multiple: $\mathrm{p}=0.007$, $\mathrm{AUC}=0.65(95 \% \mathrm{CI}=0.58-0.71))$, regardless of cognitive status. Because mood could have changed between the two assessments (they occurred at different points of the hospital stay), the multiple analyses were repeated after limiting time interval at 28, 21, and 14 days. No major improvements were noted.

Conclusion: The HADD performed poorly in elderly inpatients regardless of cognitive status. It cannot be recommended in this population for depression screening without further study.

Key words: depression, dementia, elderly, hospitalized, scale

\section{Introduction}

Depression is common in older individuals (Samaras et al., 2010). Its prevalence is approximately $13 \%$ in general and $2 \%$ for the major depression subtype (Beekman et al., 1999).

Correspondence should be addressed to: Nikolaos Samaras, MD, Department of Internal Medicine, Rehabilitation and Geriatrics, Geneva University Hospitals, Geneva, Switzerland. Chemin du Pont-Bochet 3, 1226 Thônex, Switzerland. Phone: +41795538309; Fax: +41223056115. Email: nikolassamaras@hotmail.com. Received 22 May 2012; revision requested 2 Jul 2012; revised version received 7 Aug 2012; accepted 8 Aug 2012. First published online 13 September 2012.
Depression prevalence further increases among elderly patients with dementia. Globally, up to $35 \%$ develop depression during the disease course (Aalten et al., 2005) but estimates may vary according to the dementia type: $30 \%-50 \%$ for Alzheimer's disease (AD; Zubenko et al., 2003; Burgut et al., 2006; Starkstein and Mizrahi, 2006), $52 \%-63 \%$ for vascular dementia (VD; Moretti et al., 2006), 20\%-65\% for Lewy body dementia (LBD; Burgut et al., 2006), and 20\%$45 \%$ for Parkinson disease (PD; Burgut et al., 2006). 
Depression remains underdiagnosed and undertreated in the elderly, particularly in patients with dementia (Lang et al., 2010). It is associated with faster cognitive decline and may interfere with neuropsychological testing, confounding dementia diagnosis (Lyketsos et al., 2003; Starkstein and Mizrahi, 2006). For these reasons, depression is frequently screened for in our hospital memory consultation using the depression subscale (HADD) of the Hospital Anxiety and Depression Scale (HADS). The choice of the HADD was based on the scale's simplicity, characteristics, and satisfying metric properties in younger populations. The HADS was initially developed to identify "caseness" (possible and probable) of anxiety and depression among patients in non-psychiatric wards (Zigmond and Snaith, 1983). Since then, its use has been extended to outpatient and community settings (Dunbar et al., 2000; Caci et al., 2003). The HADS is a well-accepted (Herrmann, 1997), easy-to-use, 14-item auto-questionnaire producing two 7-item subscales assessing either depression (HADD) or anxiety (HADA; Herrmann, 1997; Bjelland et al., 2002; Poole and Morgan, 2006). It is a self-assessment based on symptoms over the preceding week and is rated on a four-point Likert scale, with a maximum score of 21 on each subscale (higher scores correspond to higher disease severity; Johnston et al., 2000; Herrero et al., 2003). The HADD subscale is constructed mostly around the item of anhedonia (Poole and Morgan, 2006). Importantly, it deliberately leaves out physical indicators of psychological distress such as dizziness, lethargy, headaches, insomnia, and fatigue, to prevent interference with somatic disorders and, thus, may be particularly suited for the detection of depression in the elderly (Herrmann, 1997; Bjelland et al., 2002; Wang et al., 2006). Symptoms of severe psychopathology are also omitted to avoid the "floor effect" frequently encountered in non-psychiatric patients (Herrmann, 1997; Bjelland et al., 2002). The scale has a high internal consistency with a Cronbach's $\alpha$ coefficient of 0.7-0.9 (Herrmann, 1997; Aben et al., 2002; Bjelland et al., 2002; Herrero et al., 2003; Lowe et al., 2004; Bambauer et al., 2005; Thomas et al., 2005). Two-week test-retest reliability is also high $(r>0.80)$, demonstrating a satisfying stability of the scale (Herrmann, 1997). In younger populations, concurrent validity between HADD and other depression scales is generally satisfactory, with correlation coefficients ranging from 0.6 to 0.8 (Bjelland et al., 2002).

However, a recent study suggested a poorer performance in older populations. Helvik et al. (2011) reported a modest correlation (Spearman coefficient 0.51 ) between the HADD and the
Montgomery-Åsberg Depression Rating Scale (MADRS), although the MADRS has been well validated for the detection of depression in patients with dementia (Leontjevas et al., 2009; Helvik et al., 2011).

In a quality-control context, we retrospectively compared scores on the HADD with a psychiatric diagnosis of depression in older inpatients assessed during the same hospital stay by both a memory specialist and a psychiatrist. In this paper, we report our findings on the efficiency of the scale for depression screening, as well as the effect of dementia on the scale's performance. To our knowledge, these issues have never been addressed in this population.

\section{Methods}

This is a retrospective study comparing the scores obtained on the HADD during a memory consultation with a depression diagnosis made by the consulting psychiatrist. The study was conducted in the geriatrics ward of a university hospital as part of a quality-control project and was approved by the local ethics committee.

We reviewed the medical files of all inpatients assessed by our memory consultant between 2001 and 2006. Inclusion criteria comprised:

1. Age older than 65 .

2. Assessment by a psychiatrist during the same hospital stay and psychiatric diagnosis available.

3. Scores available on the HADD and the Clinical Dementia Rating (CDR) scale (Hughes et al., 1982).

Patients with severe dementia (CDR score of 3) were excluded.

Psychiatric and memory assessments were requested by the treating resident, according to clinical suspicion for cognitive and psychiatric disorders. The HADD was used by neuropsychologists for depression screening during the memory consultation. Because reading comprehension and visual impairments are frequent in this population, to ensure item comprehension the HADD was administered to the patient by the neuropsychologist in all of the cases. The memory specialist established a diagnosis and attributed a score of 0-3 on the CDR scale, according to the severity of dementia. The psychiatric diagnosis was established according to the Diagnostic and Statistical Manual of Mental Disorders, Fourth Edition. The same highly experienced psychiatrist evaluated all cases and the neuropsychologists performed the HADD independent of the psychiatric evaluation. The score on the HADD was compared with the 
diagnosis made by the consulting psychiatrist using the latter as a "gold standard."

The effect of dementia on the performance of the HADD was examined by dividing the total sample into three groups according to the CDR score no dementia: CDR0-0.5; early dementia: CDR1; moderate dementia: CDR2.

Because we used a convenience sample, to assess for potential selection bias we compared the group of patients included with those excluded from the study for sociodemographic and clinical variables. Finally, psychiatric and cognitive assessments occurred at different points during the patient's hospital stay. To test for a possible bias related to changes in the patient's clinical state, we repeated the analyses restricting the time difference between the two assessments to 28,21 , and 14 days.

\section{Statistical analyses}

Group comparisons were performed using the $\chi^{2}$ test, unpaired $t$-test, or Mann-Whitney $\mathrm{U}$ test, as appropriate. Simple and multiple logistic regression models were applied to predict the presence of depression diagnosis using HADD scores while adjusting for age, sex, cognitive status (CDR), and delay between assessments (determined by the absolute value of difference between the two assessment dates). Areas under the receiver operating characteristics curve (ROC) were plotted and used as measures of HADD's performance (a value of 0.5 corresponding to a useless test no different from random, and a value of 1 to the perfect test with $100 \%$ sensitivity and specificity). Areas under curves (AUC) were compared by $\chi^{2}$ tests. Specificity, sensitivity values, percentage of correctly classified cases, and most appropriate HADD cut-off score were calculated in univariate analysis for the whole group and the three subgroups (CDR0-0.5, CDR1, and CDR2). The same cut-off score was applied in multiple logistic regression analyses for the whole group to recalculate specificity, sensitivity values, and correctly classified cases. Multiple logistic regression analyses were repeated for the whole group limiting time difference between the two assessments to 28,21 , and 14 days, and for the three subgroups (CDR0-0.5, CDR1, and CDR2) without time limitation.

All analyses were performed using Stata release 12 (College Station, TX).

\section{Results}

From 2001 to 2006, 1,795 inpatients were evaluated in the memory consultation, of which 427 patients were assessed by both the memory specialist and the psychiatrist during the same hospital stay. Twelve patients were excluded because of a score of 3 on the CDR scale. Another 143 patients did not meet inclusion criteria (unavailable data for one or more of the CDR score, HADD score, and psychiatric diagnosis). A total of 272 patients were finally included in the study. We compared the included group with the 1,523 nonincluded patients. The sex distribution was identical $(\mathrm{p}=0.365)$. The age of the selected group was one year younger $(p=0.0027)$. The CDR distribution was statistically different $(\mathrm{p}<0.001)$ between the two groups (included: $24.6 \%, 45.6 \%$, and $29.8 \%$ vs. excluded: $17.3 \%, 40.2 \%$, and $42.6 \%$, for CDR: $0-0.5,1$, and 2 , respectively).

Of the included patients, 108 (39.7\%) were diagnosed with depression by the consulting psychiatrist. Fifty patients had an episode of mild depression occurring in the context of a known bipolar disorder for one patient, a recurrent depressive disorder for 12 patients, and an adjustment disorder for another 19 patients. Thirtyone patients had a depressive episode described as moderate, of which half in the context of recurrent depression. Eight patients were diagnosed with a severe depression, of which one with psychotic symptoms and three in the context of recurrent depression. Finally, for 19 patients the type and severity of the depressive episode were not specified.

Table 1 shows the demographic and clinical characteristics of the included patients. Both groups (depressed and non-depressed) were similar, except for significantly higher HADD scores $(p=0.009)$ and higher delays $(p=0.004)$ between the two assessments in the depressed group.

In a univariate analysis for the total sample, the HADD was significantly correlated with the presence of depression ( $p=0.009$; Table 2). However, sensitivity and specificity were both low (50.9\% and $69.5 \%$, respectively) using the generally accepted threshold score of 8 on the HADD (Zigmond and Snaith, 1983) and fewer than twothirds of the cases were correctly classified. In the subgroup analysis, the HADD score was correlated to depression only in the non-demented group $(\mathrm{p}=0.044$; Table 2$)$. The AUC was similar in all three groups $(\mathrm{p}=0.802$; Table 2$)$.

In a multiple regression analysis of the total sample, after adjustment for age, sex, delay, and dementia severity (CDR score), the HADD was significantly correlated with the presence of depression but performance remained modest $(\mathrm{p}=0.007, \mathrm{AUC}=0.65(95 \%$ confidence interval $(\mathrm{CI})=0.58-0.71)$; sensitivity $=62.0 \%$, specificity $=63 \%)($ Table 3$)$. Higher time intervals between psychiatric and memory assessments were significantly related to the presence of 
Table 1. Demographic and clinical characteristics $(n=272)$

\begin{tabular}{|c|c|c|c|c|}
\hline & $\begin{array}{l}\text { TOTAL SAMPLE } \\
(\mathrm{n}=272)\end{array}$ & $\begin{array}{l}\text { DEPRESSED } \\
(\mathrm{n}=108)\end{array}$ & $\begin{array}{l}\text { NON-DEPRESSED } \\
(\mathrm{n}=164)\end{array}$ & $\mathrm{p}$ \\
\hline Age, mean (SD), years & $83(5.8)$ & $83.4(5.2)$ & $82.8(5.2)$ & $0.382^{*}$ \\
\hline Male/female & $73 / 199$ & $26 / 82$ & $47 / 117$ & $0.404^{* *}$ \\
\hline CDR (\%) & & & & $0.061^{* * *}$ \\
\hline $0-0.5$ & $67(24.6)$ & $33(30.6)$ & $34(20.7)$ & \\
\hline 1 & $124(45.6)$ & $48(44.4)$ & $76(46.3)$ & \\
\hline 2 & $81(29.8)$ & $27(25)$ & $54(33)$ & \\
\hline MMSE, mean (SD) & $20.4(4.5)$ & $20.9(4.2)$ & $20.2(4.7)$ & $0.208^{*}$ \\
\hline HADS, mean (SD) & $14.5(6.4)$ & $15.6(6.6)$ & $13.8(6.2)$ & $0.022 *$ \\
\hline HADD, mean (SD) & $6.6(4)$ & $7.4(4.1)$ & $6.1(3.8)$ & $0.009^{*}$ \\
\hline HADA, mean (SD) & $7.9(3.9)$ & $8.2(4)$ & $7.7(3.9)$ & $0.294^{*}$ \\
\hline $\begin{array}{l}\text { Delay (days), mean (SD) (date } \\
\text { psychiatry-date memory) }\end{array}$ & $9.9(35.5)$ & $17.1(26.9)$ & $5.2(39.6)$ & $0.004^{*}$ \\
\hline
\end{tabular}

*Student's $t$-test; **Chi-square test; ${ }^{* * *}$ Mann-Whitney U test.

$\mathrm{CDR}=$ Clinical Dementia Rating; HADS = Hospital Anxiety and Depression Scale; HADA = Hospital Anxiety and Depression scale, Anxiety subscale; HADD $=$ Hospital Anxiety and Depression scale, Depression subscale; MMSE $=$ mini-Mental State Examination; $\mathrm{SD}=$ standard deviation.

Table 2. Performance of the HADD for depression detection: univariate analysis

\begin{tabular}{|c|c|c|c|c|c|c|}
\hline & \multicolumn{6}{|c|}{ CUT-OFF $\geq 8$} \\
\hline & $\mathrm{n}$ & $\mathrm{p}$ & AUC $(95 \% \mathrm{CI})$ & $\begin{array}{l}\text { SENSITIVITY } \\
(\%)\end{array}$ & $\begin{array}{l}\text { SPECIFICITY } \\
(\%)\end{array}$ & $\begin{array}{l}\text { CORRECTLY } \\
\text { CLASSIFIED }(\%)\end{array}$ \\
\hline Total sample & 272 & 0.009 & $0.60(0.53-0.66)$ & 50.9 & 69.5 & 62.1 \\
\hline CDR0-0.5 & 67 & 0.044 & $0.63(0.50-0.77)$ & 54.6 & 73.5 & 64.2 \\
\hline CDR1 & 124 & 0.065 & $0.59(0.49-0.69)$ & 47.9 & 67.1 & 59.7 \\
\hline CDR2 & 81 & 0.502 & $0.57(0.43-0.71)$ & 51.9 & 70.4 & 64.2 \\
\hline
\end{tabular}

$\mathrm{AUC}=$ Areas Under Curve; $\mathrm{CDR}=$ Clinical Dementia Rating; $\mathrm{CI}=$ Confidence Interval; HADD = Hospital Anxiety and Depression scale, Depression subscale.

Table 3. Performance of the HADD for depression detection. Multiple regression analysis (dementia severity according to CDR, age, sex, and delay) for the total population, and by delay limits (28, 21, and 14 days)

\begin{tabular}{|c|c|c|c|c|c|c|}
\hline & \multicolumn{6}{|c|}{$\mathrm{CUT}-\mathrm{OFF} \geq 8$} \\
\hline & $\mathrm{n}$ & $\mathrm{p}$ & AUC (95\% CI) & $\begin{array}{l}\text { SENSITIVITY } \\
(\%)\end{array}$ & $\begin{array}{l}\text { SPECIFICITY } \\
(\%)\end{array}$ & $\begin{array}{l}\text { CORRECTLY } \\
\text { CLASSIFIED }(\%)\end{array}$ \\
\hline Total sample & 272 & 0.007 & $0.65(0.58-0.71)$ & 62.0 & 60.3 & 61.0 \\
\hline$\leq 28$ days & 208 & 0.031 & $0.64(0.56-0.72)$ & 57.7 & 57.7 & 57.7 \\
\hline$\leq 21$ days & 183 & 0.067 & $0.65(0.57-0.73)$ & 56.7 & 56.9 & 56.8 \\
\hline$\leq 14$ days & 134 & 0.047 & $0.68(0.59-0.77)$ & 64.7 & 63.9 & 64.2 \\
\hline
\end{tabular}

$\mathrm{AUC}=$ Areas Under Curve; $\mathrm{CDR}=$ Clinical Dementia Rating; $\mathrm{CI}=$ Confidence Interval; HADD = Hospital Anxiety and Depression scale, Depression subscale.

depression $(p=0.010)$. However, there was no major improvement in the scale's performance when analyses were repeated after limiting the interval between the two assessments to 28,21 , and 14 days (Table 3 ).

In a multiple analysis for each CDR group after adjustment for age, sex, and delay between the two assessments, the HADD score significantly predicted depression only for the non-demented group and there was a trend toward significance for mild dementia cases (CDR0-0.5: $\mathrm{p}=0.047$, CDR1: $p=0.054$, and CDR2: $p=0.266)$. AUC were calculated on ROC curves for the three groups (CDR0-0.5: $\mathrm{AUC}=0.64(95 \% \mathrm{CI}=0.51-$ $0.78)$; $\mathrm{CDR} 1: \mathrm{AUC}=0.62(95 \% \mathrm{CI}=0.52-0.72)$; CDR2: $A U C=0.67(95 \% \mathrm{CI}=0.54-0.79))$. The 
difference among AUC was not statistically significant $(\mathrm{p}=0.837)$.

\section{Discussion}

To our knowledge, this study is the first to compare the HADD with a psychiatric assessment for depression screening in older inpatients. Moreover, it is the first to study the effect of dementia presence and severity on the scale's performance.

The most widely accepted HADD threshold for depression screening is eight (Zigmond and Snaith, 1983; Bjelland et al., 2002), for which sensitivity and specificity in the literature vary between $70 \%$ and 90\% (Aben et al., 2002; Bjelland et al., 2002; Lowe et al., 2004; Bambauer et al., 2005). In our study, the ROC curve also indicated that the above cut-off score was the most appropriate. Nevertheless, sensitivity, specificity, and accuracy were far lower than those described in younger populations, strongly suggesting that the HADD should not be used in the hospitalized elderly. Furthermore, AUCs were significantly low (0.600.65 ) compared with the value of 0.8 generally preferred in clinical tests, or those reported in younger populations (0.81-0.96; Aben et al., 2002; Bambauer et al., 2005; Rodgers et al., 2005; Poole and Morgan, 2006). Importantly, analysis by CDR group revealed only minor changes in the performance of the HADD and similar AUCS between the three groups, indicating that the modest performance of this scale in our study population was not strongly related to the presence of dementia.

Our study has several limitations. First, the depressed group included patients with various subtypes and only eight patients had severe forms of depression. Thus, we have insufficient power to report the performance of the HADD for severe depression. However, because screening tools such as the HADD are mostly used to identify cases that might otherwise go clinically undetected, its rather low sensitivity and inability to reliably detect milder cases is the most serious concern. Second, the delay between the psychiatric and neuropsychological evaluations was at times quite long and some of the depressed cases may have sufficiently improved between the two assessments to score negatively on the HADD. To take into account this potentially significant bias, we repeated the multiple regression analyses in three sub-samples including only cases with a maximum delay between assessments of 28, 21 , and 14 days. The AUC remained low in all three groups, suggesting that the relatively poor performance of the HADD was not strongly related to the above delay. Although sensitivity, specificity, and accuracy improved with shorter delays, their values also remained low, even in the group with a delay of 14 days or fewer, thus confirming that delay was not the main reason for the disappointing performance of the HADD. Finally, in keeping with a quality-control investigation, we used a convenience sample that included only cases that received both cognitive and psychiatric evaluations. This sample was not meant to be representative of all patients assessed in the memory consultation, but differences with non-included patients were minor. Moreover, some cases with very mild cognitive or depressive symptoms for whom psychiatric and memory assessments were not requested by the treating resident might have been inappropriately excluded from the study.

In our experience, the HADD did not perform well in older hospitalized patients both with or without dementia. Further prospective studies are warranted to explore this issue and to compare the HADD with other depression scales that have been developed for use in the elderly.

\section{Conflicts of interest}

None.

\section{Description of authors' roles}

Nikolaos Samaras designed the study, collected data, performed the literature review, and wrote the paper. François R. Herrmann assisted in designing the study and writing of the paper, and performed the statistical analysis. Dimitrios Samaras assisted with the data collection and writing of the paper. Pierre-Olivier Lang assisted with writing of the paper and the statistical analysis. Alessandra Canuto and Alexandre Forster assisted with writing of the paper and data collection. Henriette Hilleret performed the patients' psychiatric evaluation and assisted with writing of the paper. Gabriel Gold performed the patients' memory evaluation, participated in the design of the study and writing of the paper.

\section{Acknowledgments}

We thank our team of neuropsychologists (Lara Fazio, Malika Claire Fabjan, Delia Antille, AnneMarie Bugnon, Myriam Büchelin-Fazio, and Joëlle Emmenegger) for their contribution in passing the HADD scale.

\section{References}

Aalten, P., de Vugt, M. E., Jaspers, N., Jolles, J. and Verhey, F. R. (2005). The course of neuropsychiatric 
symptoms in dementia. Part I: findings from the two-year longitudinal Maasbed study. International fournal of Geriatric Psychiatry, 20, 523-530. doi:10.1002/gps. 1316.

Aben, I., Verhey, F., Lousberg, R., Lodder, J. and Honig, A. (2002). Validity of the beck depression inventory, hospital anxiety and depression scale, SCL-90, and Hamilton depression rating scale as screening instruments for depression in stroke patients. Psychosomatics, 43, 386-393. doi:10.1176/appi.psy.43.5.386.

Bambauer, K. Z., Locke, S. E., Aupont, O., Mullan, M. G. and McLaughlin, T. J. (2005). Using the Hospital Anxiety and Depression Scale to screen for depression in cardiac patients. General Hospital Psychiatry, 27, 275-284. doi:10.1016/j.genhosppsych.2005.03.002.

Beekman, A. T., Copeland, J. R. and Prince, M. J. (1999). Review of community prevalence of depression in later life. British Fournal of Psychiatry, 174, 307-311. doi:10.1192/bjp.174.4.307.

Bjelland, I., Dahl, A. A., Haug, T. T. and Neckelmann, D. (2002). The validity of the Hospital Anxiety and Depression Scale. An updated literature review. Fournal of Psychosomatic Research, 52, 69-77. doi:10.1016/S0022-3999(01)00296-3.

Burgut, F. T., Benaur, M. and Hencliffe, C. (2006). Late-life depression: a neuropsychiatric approach. Expert Review of Neurotherapeutics, 6, 65-72. doi:10.1586/14737175.6.1.65.

Caci, H., Bayle, F. J., Mattei, V., Dossios, C., Robert, P. and Boyer, P. (2003). How does the Hospital and Anxiety and Depression Scale measure anxiety and depression in healthy subjects? Psychiatry Research, 118, 89-99. doi:10.1016/S0165-1781(03)00044-1.

Dunbar, M., Ford, G., Hunt, K. and Der, G. (2000). A confirmatory factor analysis of the Hospital Anxiety and Depression scale: comparing empirically and theoretically derived structures. British fournal of Clinical Psychology, 39, 79-94. doi:10.1348/014466500163121.

Helvik, A. S., Engedal, K., Skancke, R. H. and Selbæk, G. (2011). A psychometric evaluation of the Hospital Anxiety and Depression Scale for the medically hospitalized elderly. Nordic fournal of Psychiatry, 65, 338-344. doi:10.3109/08039488.2011.560684.

Herrero, M. J., Blanch, J., Peri, J. M., De Pablo, J., Pintor, L. and Bulbena, A. (2003). A validation study of the hospital anxiety and depression scale (HADS) in a Spanish population. General Hospital Psychiatry, 25, 277-283. doi:10.1016/S0163-8343(03)00043-4.

Herrmann, C. (1997). International experiences with the Hospital Anxiety and Depression scale - a review of validation data and clinical results. Fournal of Psychosomatic Research, 42, 17-41. doi:10.1016/S0022-3999(96)00216-4.

Hughes, C. P., Berg, L., Danziger, W. L., Coben, L. A. and Martin, R. L. (1982). A new clinical scale for the staging of dementia. British fournal of Psychiatry, 140, 566-572. doi:10.1192/bjp.140.6.566.

Johnston, M., Pollard, B. and Hennessey, P. (2000). Construct validation of the hospital anxiety and depression scale with clinical populations. Fournal of Psychosomatic
Research, 48, 579-584. doi:10.1016/S00223999(00)00102-1.

Lang, P. O. et al. (2010). Potentially inappropriate prescribing including under-use amongst older patients with cognitive or psychiatric co-morbidities. Age and Ageing, 39, 373-381. doi:10.1093/ageing/afq031.

Leontjevas, R., van Hooren, S. and Mulders, A. (2009). The Montgomery-Asberg Depression Rating Scale and the Cornell Scale for Depression in Dementia: a validation study with patients exhibiting early-onset dementia. American fournal of Geriatric Psychiatry, 17, 56-64. doi:10.1097/JGP.0b013e31818b4111.

Lowe, B. et al. (2004). Comparative validity of three screening questionnaires for DSM-IV depressive disorders and physicians' diagnoses. Fournal of Affective Disorders, 78, 131-140. doi:10.1016/S0165-0327(02)00237-9.

Lyketsos, C. G. et al. (2003). Treating depression in Alzheimer disease: efficacy and safety of sertraline therapy, and the benefits of depression reduction: the DIADS. Archives of General Psychiatry, 60, 737-746. doi:10.1001/archpsyc.60.7.737.

Moretti, R., Torre, P., Antonello, R. M. and Cazzato, G. (2006). Behavioral alterations and vascular dementia. Neurologist, 12, 43-47. doi:10.1097/01.nrl.0000186806.54314.e8.

Poole, N. A. and Morgan, J. F. (2006). Validity and reliability of the Hospital Anxiety and Depression Scale in a hypertrophic cardiomyopathy clinic: the HADS in a cardiomyopathy population. General Hospital Psychiatry, 28, 55-58. doi:10.1016/j.genhosppsych.2005.08.004.

Rodgers, J., Martin, C. R., Morse, R. C., Kendell, K. and Verrill, M. (2005). An investigation into the psychometric properties of the Hospital Anxiety and Depression scale in patients with breast cancer. Health and Quality of Life Outcomes, 3, 41. doi: 10.1186/1477-7525-3-41.

Samaras, N., Rossi, G., Giannakopoulos, P. and Gold, G. (2010). Vascular depression. An age-related mood disorder. European Geriatric Medicine, 1, 220-225. doi:10.1016/j.eurger.2010.05.006.

Starkstein, S. E. and Mizrahi, R. (2006). Depression in Alzheimer's disease. Expert Review of Neurotherapeutics, 6, 887-895. doi:10.1586/14737175.6.6.887.

Thomas, B. C. et al. (2005). Reliability and validity of the Malayalam Hospital Anxiety and Depression Scale (HADS) in cancer patients. Indian fournal of Medical Research, 122, 395-399.

Wang, W., Lopez, V. and Martin, C. R. (2006). Structural ambiguity of the Chinese version of the Hospital Anxiety and Depression scale in patients with coronary heart disease. Health and Quality of Life Outcomes, 4, 6. doi:10.1186/1477-7525-4-6.

Zigmond, A. S. and Snaith, R. P. (1983). The hospital anxiety and depression scale. Acta Psychiatrica Scandinavica, 67, 361-370. doi:10.1111/j.1600-0447.1983. tb09716.x.

Zubenko, G. S. et al. (2003). A collaborative study of the emergence and clinical features of the major depressive syndrome of Alzheimer's disease. American fournal of Psychiatry, 160, 857-866. doi:10.1176/appi.ajp.160.5.857. 